\title{
Nanoscale holographic tomography of heart tissue with x-ray waveguide optics
}

M. Reichardt, J. Frohn, M. Töpperwien, J.-D. Nicolas, A. Markus, et al.

M. Reichardt, J. Frohn, M. Töpperwien, J.-D. Nicolas, A. Markus, F. Alves, T. Salditt, "Nanoscale holographic tomography of heart tissue with x-ray waveguide optics," Proc. SPIE 10391, Developments in X-Ray Tomography XI, 1039105 (25 September 2017); doi: 10.1117/12.2276648

EDent: SPIE Optical Engineering + Applications, 2017, San Diego, California, United States 


\title{
Nanoscale holographic tomography of heart tissue with x-ray waveguide optics
}

\author{
M. Reichardta ${ }^{\mathrm{a},}$, J. Frohn ${ }^{\mathrm{a},}$,, M. Töpperwien ${ }^{\mathrm{a}}$, J.-D. Nicolas ${ }^{\mathrm{a}}$, A. Markus ${ }^{\mathrm{b}}$, F. Alves ${ }^{\mathrm{b}, \mathrm{c}}$, and \\ T. Salditt ${ }^{\mathrm{a}}$ \\ anstitute for X-Ray Physics, Georg-August-University Göttingen, Friedrich-Hund-Platz 1, \\ Göttingen 37077, Germany. \\ ${ }^{b}$ Max Planck Institute for Experimental Medicine, Herman-Rein-Str. 3, Göttingen 37077, \\ Germany. \\ ${ }^{c}$ Clinic of Haematology and Medical Oncology, University Medical Center Göttingen, Robert \\ Koch Strasse 40, Göttingen 37075, Germany.
}

\begin{abstract}
In this work we present x-ray phase-contrast tomography of heart tissue from mouse, combining computed tomography (CT) scans with laboratory and synchrotron radiation. The work serves as a proof-of-concept that the cyto-architecture and in particular the myofibril orientation can be assessed in three dimensions (3D) by phase-contrast CT. We demonstrate the synergistic use of laboratory $\mu$-CT and of the high resolution synchrotron setup based on waveguide optics. Details on preparation, instrumentation and analysis are given, as a state of the art reference for heart tissue tomography, and as a starting point for further progress.
\end{abstract}

Keywords: X-ray tomography, phase contrast, in-line holography, 3D virtual histology, heart tissue

\section{INTRODUCTION}

Heart contractility as one of the most important physiological functions relies on an intricate molecular- and cyto-architecture. Classical diffraction studies on muscle (both for skeletal and cardiac muscle) have contributed to the standard model of the sarcomere as the basic structural unit of the myofibrils which make up muscle fibers. Conventional histology, on the other hand, enables the visualisation of the cyto-architecture, however, only in two dimensions (2D). What is lacking today is a method to assess the tissue structure in full 3D, including in particular the local orientation, and the entire three-dimensional assembly of myofibrils.

Scanning x-ray diffraction is one option to probe molecular orientation of heart tissue, combining the required real space resolution with molecular sensitivity by diffraction. In addition to the sarcomere, structure parameters of the myocardial collagen matrix in heart tissue are of interest for heart pathologies. However, there are two major challenges: First, the scanning technique is prohibitively slow for 3D studies, since diffraction tomography requires scanning of three degrees of freedom. Secondly, the dose involved in scanning diffraction is typically also very high. In practice, this approach therefore is restricted to $2 \mathrm{D}$ slices.

In this work, we want to explore to which end full field x-ray tomography can also reveal details of the heart muscle cyto-architecture, in view of a full 3D histological representation at a resolution higher than that of optical microscopy. Such 3D data of the cyto-architecture should then be combined with conventional histology as well as scanning x-ray diffraction images to probe the molecular structure. In particular, we want to verify whether the contrast of fixed but unstained tissue is high enough for this purpose. Further, the goal of this work is to delineate phase-contrast tomography at laboratory $\mu$-CT setups from high resolution synchrotron tomography. For both modalities, we present novel approaches to instrumentation and optics, which we have developed for the purpose of 3D histological studies by phase-contrast x-ray tomography, both at the nano- and micro-scale.

* These authors contributed equally to the work.

Further author information:

T. Salditt, Corresponding author, Tel.: (+49)551-399427, Email: tsaldit@gwdg.de

Developments in X-Ray Tomography XI, edited by Bert Müller, Ge Wang, Proc. of SPIE Vol. 10391, 1039105 - (c) 2017 SPIE · CCC code: 0277-786X/17/\$18 - doi: 10.1117/12.2276648 


\section{METHODS AND EXPERIMENTAL REALIZATION}

\subsection{Sample preparation}

A heart was dissected from a 7 months old healthy female wildtype C57BL/6 mouse. The heart, sketched in Fig. 1a, was fixed overnight in $10 \%$ formalin solution, dehydrated using an increasing ethanol series and subsequently embedded in a block of paraffin, shown in Fig. 1b. Biopsies were taken with a needle of $1 \mathrm{~mm}$ diameter (GE Healthcare Life Science, Chalfont St. Giles, UK), transferred to a polyimide tube (Goodfellow Cambridge limited, Huntingdon, England) and mounted on a brass pin (HUBER Diffraktionstechnik GmbH \& Co. KG, Rimsting, Germany) using two-component glue (see Fig. 1c). For this specific heart, three biopsies were taken. In order to screen the quality of the biopsy punches, in-house tomography was carried out using the setup described in section 2.3. An image of one biopsy placed in the sample stage at the in-house setup is shown in Fig. 1d.

The biopsy with highest quality (no paraffin fractures) was then measured at beamline P10 at DESY in Hamburg using the Göttingen instrument for nanoscale imaging with x-rays (GINIX) to visualize the 3D cytoarchitecture. $^{2-7}$

\subsection{Alignment of a tomographic setup}

Before a tomographic measurement can be recorded, several alignment steps have to be carried out in order to optimize the final quality of the tomogram.

First, the pitch angle $\alpha$ between the tomographic and optical axis, shown in Fig. 2a, has to be minimized. To this end, two images at different positions $x_{1}$ along the optical axis are recorded. The pitch angle $\alpha$ is given by

$$
\alpha=\arctan \frac{\Delta z}{\Delta x}
$$

where $\Delta z$ is the change in height and $\Delta x$ the change along the optical axis for one feature in the sample. Due to different magnifications of both images the observation of height changes may be challenging, as both effects interfere. However, if the rotation axis is perfectly perpendicular to the optical axis, the sample feature which lies directly on the optical axis shows no lateral changes between the two recorded images. This can be used for alignment instead by iteratively refining the pitch angle until translations along $z$ are minimized and differences between the two recorded images are solely caused by magnification effects.

As a second step of alignment, the rotation axis has to be centered in the field of view of the detector. This is achieved by recording a standard TEM copper grid at $0^{\circ}$ and at $180^{\circ}$ of the current tomographic axis. By horizontally flipping one image and subtracting both images the residual shift of the rotation axis is given by the
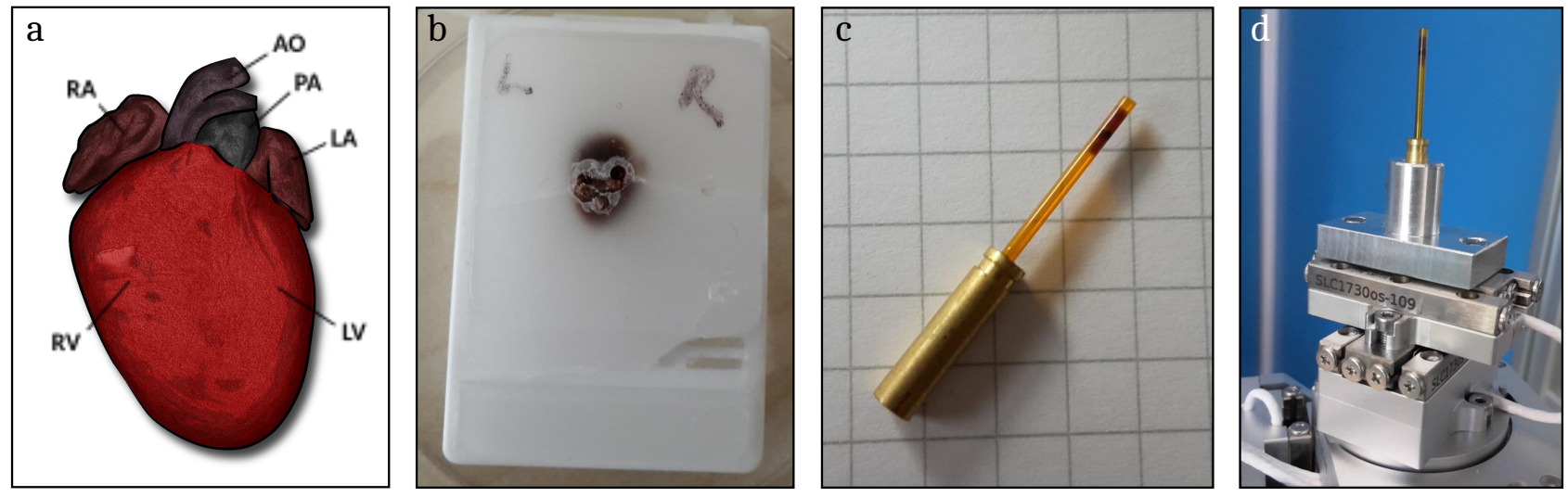

Figure 1. Sample preparation. (a) Sketch of a healthy heart, ${ }^{1}$ dissected from a 7 months old female wildtype C57BL $/ 6$ mouse, chemically fixed, dehydrated and embedded in paraffin. RA, right artery; LA, left artery; AO, aorta; PA, pulmonary artery; LV, left ventricle; RV, right ventricle. (b) Paraffin block, from which three biopsies of different regions were taken, inserted in (c) the poylimide tube shown and scanned by in-house $\mu$-CT scans to screen for intactness and sample quality . (d) An image of one biopsy placed in the sample stage at the in-house setup. 

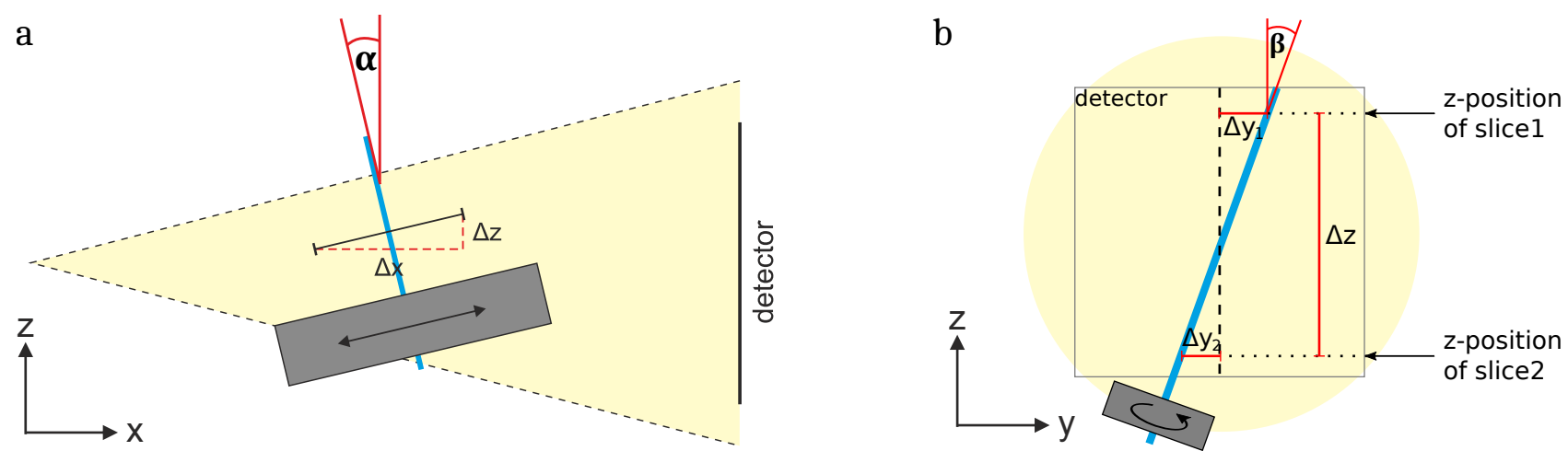

Figure 2. Tomographic alignment. (a) To align the rotation axis perpendicular to the optical axis, the pitch angle $\alpha$ has to be adjusted. For this purpose two images at different distances to the source are recorded by a translation above the rotation stage, and the image shift along $z$ is determined. The pitch angle $\alpha=\arctan \frac{\Delta z}{\Delta x}$ is then corrected by a tilt stage below the rotation stage. ${ }^{8}$ (b) Eventual misalignment of the roll angle $\beta$ is accounted for by image rotation after data acquisition. To this end, two slices are reconstructed and the position of the tomographic axis is refined.

shift needed to bring both into line. ${ }^{8}$ This procedure is automatized using a custom-written MATLAB script. ${ }^{9}$ Finally, the effective pixel size $p_{\text {eff }}$ and consequently the exact source-to-detector distance $x_{02}$ have to be determined. To this end, the test target is moved along $y$ by a defined distance $\Delta y_{o}$. By comparing $\Delta y_{o}$ to the shift of the image on the detector, given in number of pixels $\Delta y_{d}$, the effective pixel size is given by

$$
p_{\text {eff }}=\frac{\Delta y_{o}}{\Delta y_{d}} .
$$

Assuming a well-known source-to-sample distance $x_{1}$ the source-to-detector distance $x_{02}$ can be calculated using

$$
x_{02}=x_{1} \cdot M=x_{1} \cdot \frac{p}{p_{\mathrm{eff}}},
$$

where $M$ is the magnification and $p$ the actual detector pixel size. This procedure can again be automatized by using a custom-written MATLAB script. Note that at the GINIX setup, the calibration of $x_{1}$ can be achieved by the motorized on-axis optical microscope, with a reference position at the KB focal plane can be used at the GINIX setup. A misalignment of the roll angle $\beta$ of the tomographic axis, which describes the rotation around the optical axis as shown in Fig. 2b, leads to a height change of sample features during rotation which causes artifacts in the tomographic reconstruction. However, this was not corrected for during the experimental alignment since $\beta$ can be more accurately determined during the reconstruction and misalignment can be compensated by simply rotating all projections accordingly. To find out the roll angle of the rotation axis two slices at different heights $z$ within the recorded volume are reconstructed and the shift $\Delta y$ of the rotation axis with respect to the center of the detector is optimized for each slice independently. In the case $\beta=0^{\circ}$ the shifts determined at the different $z$ positions are equal while for $\beta \neq 0^{\circ}$ they differ. The difference between the determined shifts can then be used for the calculation of the roll angle via

$$
\beta=\arctan \frac{\Delta y_{1}-\Delta y_{2}}{\Delta z},
$$

where $\Delta z$ is the height difference of the two reconstructed slices.

\subsection{Laboratory experiment}

Setup To use beamtime at the synchrotron facility efficiently, it is of advantage to have prior information about the shape, structure and preparation quality of samples or sample candidates. Apart from screening the sample quality and intactness, it is also important to visualize larger FOVs at moderate resolution, in order to best 'place' the high resolution synchrotron scan. To this end, we have built and commissioned an in-house laboratory $\mu$-CT setup shown in Fig. 3a, well suited for absorption and phase-contrast tomography based on free-space propagation of small tissue samples. In this study, we used this setup to narrow down the choice 
of the most relevant sample and field of view for the subsequent synchrotron beamtime. The setup consists of three components illustrated in Fig. 3a: x-ray source, sample tower and detector tower. The x-ray source is a rotating copper anode (Rigaku, Tokyo, Japan) with an elliptical electron focal spot size of $700 \times 70 \mu \mathrm{m}^{2}$ at the anode surface, operated at $U=40 \mathrm{keV}$ and $I=30 \mathrm{~mA}$. At a take-off angle of $6^{\circ}$ the x-ray spot is circular with a diameter of $\mathrm{FWHM}_{\text {source }}=70 \mu \mathrm{m}$. The emitted x-rays leave the vacuum chamber through a $0.2 \mathrm{~mm}$ thick beryllium window in a cone-beam geometry. The x-ray energy spectrum has the characteristic copper- $K_{\alpha}$ peak at $8.048 \mathrm{keV}$ and a maximum energy at $40 \mathrm{keV}$ resulting from the bremsstrahlung. The partial coherence needed for propagation-based phase-contrast imaging is achieved by the small focal spot with respect to the long distance $x_{1}$, which describes the distance between the focal spot $S$ and sample position. The sample is mounted on a tower, equipped with 5 motorized stages for a precise and variable alignment. It consists of two translational stages ( $\mathrm{y}$ and $\mathrm{z}$ ) for the alignment of the tomographic axis, one rotational stage required for tomography and two additional translations above the rotation to bring the sample's region of interest into the field of view. The detector is placed at a distance $x_{2}$ behind the sample and is mounted on a tower consisting of 4 manual positioners ( 1 translation +3 rotations). A precise alignment of the detector perpendicular to the optical axis is pursued while the mounting is kept as stable as possible to avoid loss of resolution caused by vibrations. Sample tower and detector tower are placed on an extra granite cube to reduce vibrations. A cone-beam setup for propagation-based phase-contrast imaging can be operated using two different geometries, in order to optimize resolution for given source spot size and detector pixel size. ${ }^{10,11}$ The optimum choice of geometric parameters depends on $\mathrm{FWHM}_{\text {source }}$ and the point spread function (PSF) of the detector $\mathrm{FWHM}_{\text {det }}$, which is bounded
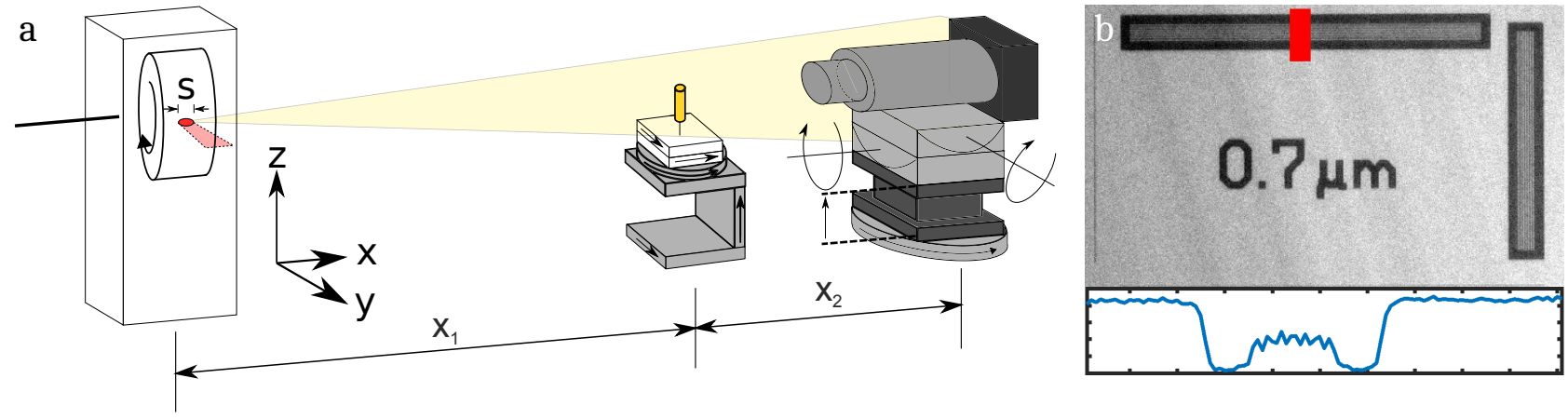

C

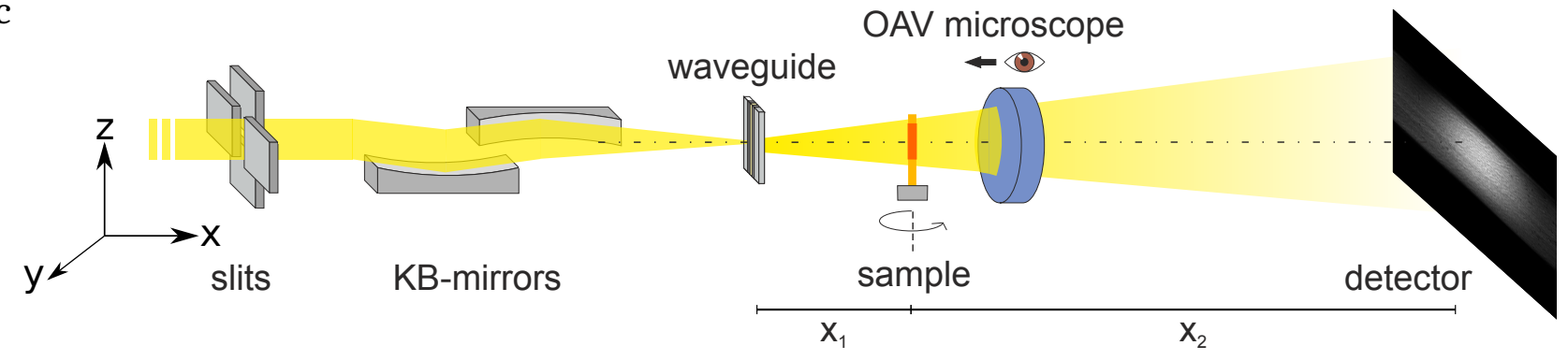

Figure 3. Tomography setups for laboratory $\mu$-CT and synchrotron CT (GINIX/ P10 beamline). (a) Schematic of the in-house setup consisting of three components with translational and rotational degrees of freedom: (i) rotating copper anode creating the x-rays (yellow) at the focal spot $S$ of the focused electron beam (red) (ii) sample tower including 5 motorized stages for a precise alignment of the tomographic axis and the ability to change the ROI of the sample, (iii) detector tower comprising 4 manual stages for a stable detector alignment perpendicular to the optical axis. (b) Projection of the test pattern obtained with $t=180 \mathrm{~s}$ exposure time. An intensity profile of the red line through the lines and spaces test pattern is plotted below, averaged over a 30 pixel line width. The $700 \mathrm{~nm}$ lines and spaces pairs are resolved at the in-house setup. (c) Schematic of the GINIX setup at the P10 beamline (DESY, Hamburg). ${ }^{3}$ The beam was focused to a spot size of about $350 \mathrm{~nm}$ (vert.) x $290 \mathrm{~nm}$ (horz.) by two KB-mirrors onto the front side of a planar waveguide with a guiding layer of $82 \mathrm{~nm}$ thickness resulting in a secondary source spot of $18 \mathrm{~nm}$ in height. The sample was mounted on a motorized sample stage and was monitored with a on axis microscope (OAV). Images were acquired with a fiber-coupled scintillator based sCMOS camera. 
from below by the pixel size of the detector. In the case $x_{1} \ll x_{2}$, the magnifying geometry, a high optical magnification $M$ yields a small effective pixel size and hence a high resolution. For $\mathrm{FWHM}_{\text {source }}<\mathrm{FWHM}_{\text {det }}$ this geometry should be used as we did at the synchrotron. The resolution is then limited by the focal spot

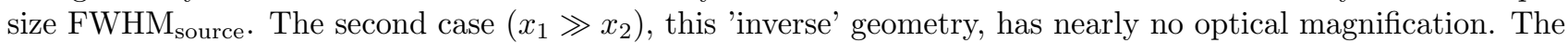
resolution is therefore limited by the detector point spread function $\mathrm{FWHM}_{\text {det }}$. Due to the comparably large focal spot size of the rotating anode, our laboratory setup is built in the inverse geometry $\left(x_{1} \gg x_{2}\right)$, and the high resolution detector XSight (Rigaku, Prague, Czech Republic) with a lens-coupled single crystal scintillator CCD camera is used. The pixel size is $\mathrm{FWHM}_{\text {det }}=0.54 \mu \mathrm{m}$ with a field of view of $1.8 \times 1.4 \mathrm{~mm}^{2}$. With $x_{1}=465 \mathrm{~mm}$ and $x_{2}=6 \mathrm{~mm}$, the $700 \mathrm{~nm}$ lines and spaces of the Wo test pattern RT RC-02 (JIMA, Tokyo, Japan) are resolved as presented in Fig. 3b.

Data acquisition and reconstruction For the tomographic scan, 25 darkfield images, 25 flatfield images and 1000 projections over $180^{\circ}$ were recorded with an exposure time of $t=40 \mathrm{~s}$ each. The projections were emptybeam corrected and binned by a factor of 2 in order to achieve a higher signal to noise ratio. Phase retrieval of each projection was then performed by applying the Bronnikov-Aided-Correction algorithm (BAC),${ }^{12}$ described in the next paragraph. Subsequently, the tomographic reconstrucion was carried out using the (cone-beam) filtered back-projection implementation of the ASTRA toolbox. ${ }^{13,14}$ All acquisition and reconstruction parameters are summarized in Tab. 1.

Phase retrieval Phase-contrast imaging is used for weakly absorbing objects to obtain sufficient contrast for high resolution x-ray imaging. To remove interference effects such as edge enhancement it is necessary to reconstruct the phase of the object using a phase-retrieval algorithm. In this study we used the Bronnikov-aided correction (BAC) algorithm which gives optimal results for laboratory-based phase-contrast imaging in the direct contrast regime. ${ }^{15}$ Reconstruction is carried out in two steps. First, the modified Bronnikov algorithm (MBA) ${ }^{16}$ is used to reconstruct an approximate phase image $\phi^{\dagger}\left(\mathbf{r}_{\perp}\right)$ with $\mathbf{r}_{\perp}=(y, z)$ from the projections using the absorption-dependent regularization parameter $\alpha$. As this algorithm assumes a pure phase object, it leads to blurred reconstructions due to residual absorption. Therefore, the second step of the BAC is used to sharpen the images again by assuming a weakly absorbing object and reconstructing the intensity distribution in the object plane from the approximated phase $\phi^{\dagger}\left(\mathbf{r}_{\perp}\right)$ via

$$
I_{a}\left(\mathbf{r}_{\perp}\right)=\frac{I\left(\mathbf{r}_{\perp}, \Delta x\right)}{\left(1-\gamma \nabla_{\perp}^{2} \phi^{\dagger}\left(\mathbf{r}_{\perp}\right)\right)},
$$

where $\gamma$ is a second $\alpha$-dependent regularization parameter.

\subsection{Synchrotron experiment}

Setup A high resolution tomogram of the sample was recorded at the GINIX setup which is available at the P10 beamline of the storage ring PETRA III at DESY in Hamburg. ${ }^{2,8,17}$ The schematic design of the setup is shown in Fig. 3c. The beam is created by an undulator and monochromatized by a polished $\mathrm{Si}(111)$ channel-cut crystal monochromator that limits the bandwidth of the x-rays to $\frac{\Delta \lambda}{\lambda}=10^{-4}$. The beam size is defined by a pair of slits before two Kirkpatrick-Baez (KB) mirrors focus the radiation to a spot size of about $350 \mathrm{~nm} \times 290 \mathrm{~nm}$. A good performance of the mirrors is achieved at an energy range between $E_{\min }=6 \mathrm{keV}$ and $E_{\max }=15 \mathrm{keV}$. For the tomography of the heart biopsy, an energy of $13.8 \mathrm{keV}$ was chosen. At the focal spot of the KB-mirrors an $\mathrm{x}$-ray waveguide (Mo/C/Mo planar waveguided) is placed and can be aligned by a parallel kinematic robot in a hexapod configuration. The coherence of the exit wave is increased by filtering of modes in the waveguide, and the beam is further confined. Its profile is smoothened and can be approximated by a Gaussian ${ }^{3,18}$ which reduces artifacts in the empty-beam correction caused by the structured illumination of the pure KB beam. ${ }^{19}$ The sample is mounted on a motorized sample stage with linear $\mathrm{x}-, \mathrm{y}-, \mathrm{z}$-positioners and rotations for three dimensions. Additionally, the rotation stage is based on another set of three linear positioners to align the center of rotation. Furthermore, an on-axis video microscope (OAV) equipped with a network camera can be moved in the beam to prealign the sample's region of interest in the x-ray beam. The projections are recorded by a fiber-coupled scintillator-based sCMOS camera (2048 x 2048 pixels, Photonic Science, Sussex, UK) with a custom $15 \mu \mathrm{m}$ gadox scintillator and a pixel size of $6.5 \mu \mathrm{m}$ which is placed approximately $5 \mathrm{~m}$ behind the sample. 
Table 1. Acquisition and reconstruction parameters of the laboratory setup and the synchroton experiment performed at beamline P10 (PETRA III, DESY)

\begin{tabular}{|l|l|l|}
\hline parameters & laboratory & synchrotron \\
\hline \hline energy $E$ & $0-40 \mathrm{keV}\left(\mathrm{K}_{\alpha} 8.048 \mathrm{keV}\right)$ & $13.8 \mathrm{keV}$ \\
\hline source to detector distance $x_{1}+x_{2}$ & $465 \mathrm{~mm}$ & $5049 \mathrm{~mm}$ \\
\hline focal spot size $S$ & $70 \times 70 \mu \mathrm{m}^{2}($ circ $)$ & $290 \times 18 \mathrm{~nm}^{2}$ \\
\hline pixel size $p$ & $0.54 \mu \mathrm{m}$ & $6.5 \mu \mathrm{m}$ \\
\hline defocus distances $x_{1}$ & $459 \mathrm{~mm}$ & $145 \mathrm{~mm}, 150 \mathrm{~mm}, 160 \mathrm{~mm}, 180 \mathrm{~mm}$ \\
\hline magnification $M$ & 1.02 & $34.9,33.7,31.6,28.1$ \\
\hline effective pixel size $p_{\text {eff }}$ & $0.53 \mu \mathrm{m}$ & $187.4 \mathrm{~nm}, 193.9 \mathrm{~nm}, 206.8 \mathrm{~nm}, 232.7 \mathrm{~nm}$ \\
\hline number of projections over $180^{\circ}$ & 1000 & 1001 \\
\hline exposure time $t$ & $40 \mathrm{~s}$ & $0.1 \mathrm{~s}$ \\
\hline number of flatfield images & $25($ only before) & 50 \\
\hline number of darkfield images & 25 & 25 \\
\hline \hline phase retrieval parameters & $\alpha=0.0663$ (MBA) & $\beta / \delta=1 / 20$ \\
\hline & $\gamma=0.156$ (BAC) & $\alpha_{1}=\alpha_{2}=0$ \\
\hline
\end{tabular}

Data acquisition By comparing images of the on-axis video microscope with reconstructions from the inhouse measurement the region of interest was moved to the field of view. Due to the use of a 1D (planar) waveguide, the illumination on the detector is highly anisotropic in the horizontal direction, as shown in Fig. 3c. The illuminated area of $1600 \times 836$ pixels corresponds to an effective field of view of about $300 \times 150 \mu \mathrm{m}^{2}$.

After the alignment of the sample, tomographic datasets were recorded at four source-to-sample distances $x_{1} \in$ $\{145,150,160,180\} \mathrm{mm}$. Choosing four defocus distances has proven to be an optimum choice for the application of the CTF-based phase-retrieval algorithm presented below. ${ }^{4,20}$ For every defocus distance 1001 projections over $180^{\circ}$ with an exposure time of $0.1 \mathrm{~s}$ were acquired. Additionally, 50 flatfield images were taken before and after each tomogram. For darkfield correction 25 images without beam were recorded. All experimental parameters are summarized in Tab. 1.

Phase retrieval For large propagation distances and hence small Fresnel numbers, as in this experiment, phase contrast is visible in the form of multiple fringes that appear around an object. One single-step method for phase reconstruction is based on the contrast transfer function (CTF), $, 4,21,22$ which is valid for weakly absorbing objects with a slowly varying phase. By additionally assuming a homogeneous and optically thin object with a constant $\frac{\beta}{\delta}$-ratio $(\beta$ and $\delta$ as defined by the complex index of refraction $n=1-\delta+i \beta)$, the phase can be reconstructed using all four propagation distances via ${ }^{22}$

$$
\phi\left(\mathbf{r}_{\perp}\right)=\mathcal{F}^{-1}\left[\frac{\sum_{m=1}^{N} \mathcal{F}\left[I\left(\mathbf{r}_{\perp}, x_{1, m}\right)\right] \cdot\left(\sin \chi_{m}+\frac{1}{\kappa} \cos \chi_{m}\right)}{\sum_{m=1}^{N} 2 \cdot\left(\sin \chi_{m}+\frac{\beta}{\delta} \cos \chi_{m}\right)^{2}+\alpha\left(\mathbf{k}_{\perp}\right)}\right],
$$

where $I\left(\mathbf{r}_{\perp}\right)$ denotes the empty-beam corrected intensity distribution, $\mathcal{F}$ the lateral Fourier transform with the coordinates $\mathbf{k}_{\perp}=\left(k_{y}, k_{z}\right)$ and $\chi_{m}$ is given by $\chi_{m}=\frac{\lambda x_{1, m}\left|\mathbf{k}_{\perp}\right|^{2}}{4 \pi}$. In order to compensate for zero-crossings of the denominator, a frequency-dependent regularization parameter $\alpha\left(\mathbf{k}_{\perp}\right)$ is used, which is defined as in previous works. ${ }^{4,20}$ Note that the non-negligible absorption provides a 'natural' regularization for small spatial frequencies so that only the larger spatial frequencies have to be further regularized. 


\section{RESULTS}

\subsection{In-house results}

In view of the experiment on cardiac tissue at the synchrotron, samples were pre-characterized at the in-house $\mu$-CT setup in order to judge the preparation quality and identify regions of interest within the sample. In this case, special care was taken to select a specimen that contained the least amount of preparation artifacts such as fissures. Fig. 4 visualizes the in-house results of such a sample. One empty-beam corrected projection of the sample (top left) and its phase reconstructed image using the BAC algorithm (bottom right), separated by the diagonal straight line, are presented in Fig. 4a. In the empty-beam corrected projection the edge enhancement is clearly visible at the polyimide tube. As can be seen from the intensity of the reconstructed phase image this effect is corrected. According to this projection, the sample seems intact and homogeneous except for the upper region, showing elongated structures. The arrow indicates the position of the transverse slice through the volume (straight horizontal line) shown in Fig. 4c. A longitudinal slice $(x z)$ through the reconstructed 3D volume is shown in Fig. 4b. The dark regions can be identified as the heart tissue while the bright regions indicate paraffin filling out voids between muscle fibers. The thick black vertical lines are the polyimide tube. The slice
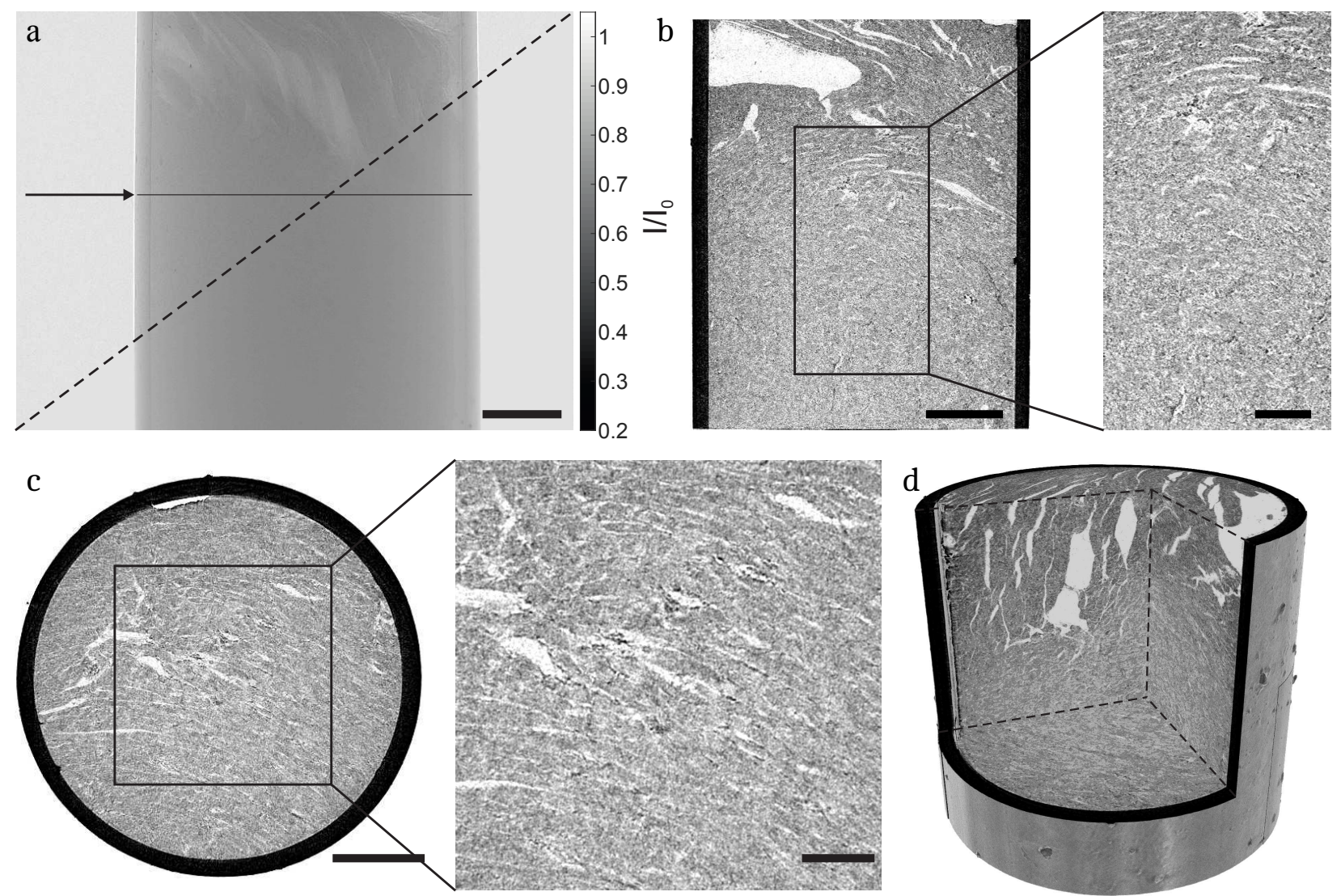

Figure 4. In-house tomography results of heart tissue in a polyimide tube. (a) Upper left: An empty-beam corrected projection obtained with an exposure time of $t=40 \mathrm{~s}$. Edge enhancement is visible at the polyimide tube edge. Lower right: Phase reconstructed image obtained after application of the BAC algorithm with parameters $\alpha=0.0663$ and $\gamma=0.156$. Both images are binned by a factor of 2 . The sample seems homogeneous in the lower part and elongated structures are visible in the upper part. Arrow and solid line indicate the height of the transverse slice in (c). (b) Longitudinal slice $(x z)$ through the reconstructed 3D volume with a magnified area of interest. The lower part of the slice shows unresolved homogeneous tissue while in the upper part damaged structure in form of ruptures filled with paraffin can be recognized. (c) Transverse slice $(x y)$ through the reconstructed 3D volume with a magnified area of interest. Fiber orientation from left to right is resolved to some extent. (d) View into the 3D reconstructed volume of the rendered data by Avizo showing internal structures. Scale bars: $250 \mu \mathrm{m}$ and insets $100 \mu \mathrm{m}$ 
shows damaged heart tissue in form of ruptures at the top and a widely homogeneous unresolvable pattern in the lower part. This impression of smaller substructures, which are only partially resolved, is emphasized by the magnified inset of a subregion of the slice. However, the inset also shows more structured and curved regions at the top possibly indicating orientation of the muscle fibers.

The transverse slice $(x y)$ in Fig. 4c shows some damages as well. Additionally to the fissures within the tissue, some artifacts caused by the insertion of the heart tissue into the polyimide tube can be recognized in the form of air between the tissue and the tube (top of the slice). Further, some fiber orientation in horizontal direction can be recognized, an impression which is accentuated in the magnified region shown in the inset on the right. A 3D volume rendering of the data can be seen in Fig. 4d with a cubic cut into the volume showing the internal structure of the sample. The data was visualized with the software Avizo (FEI Visualization Sciences Group, Burlington, USA). As the lower region of the sample consists of more homogeneous tissue without preparation artifacts it is subject to further experiments at the synchrotron. We conclude, that heart tissue samples can be successfully pre-characterized in $3 \mathrm{D}$, with a sufficient resolution throughout, using $\mu$-CT instrumentation with permanent accessibility. Ruptures filled with paraffin can be clearly recognized while other components such as erythrocytes, nuclei and single myofibers are not clearly resolved. To unambiguously visualize these (sub-)structures, a high resolution setup such as the GINIX instrument is required.

\subsection{Synchrotron results}

As for the in-house measurement, data reconstruction was performed using MATLAB. ${ }^{9}$ The projections of all distances were reconstructed using the CTF approach (see fig. 5a and b), described in Sec. 2.4. To account for different magnifications at the different defous distances, the normalized projections were rescaled to the smallest pixel size and aligned to each other by cross correlation. Prior to tomographic reconstruction, a sinogram-based ring removal was performed ${ }^{10,23}$ and the roll angle $\beta$ was corrected as explained in Sec. 2.2. Finally, all slices were reconstructed using the standard inverse Radon transform with a Ram-Lak filter. In Fig. $5 \mathrm{c}-\mathrm{f}$ the results of the tomographic reconstruction are shown. In comparison to the in-house results the resolution of the reconstructed slices is clearly higher as sub-structures like the cardiac muscle fibers with a diameter of about $10 \mu \mathrm{m}$ and single erythrocytes with a diameter of 5-8 $\mu \mathrm{m}$ and a thickness of around $2 \mu \mathrm{m}$ as well as aggregates are visible. Due to the high iron concentration of the hemoglobin in the cytoplasm of the erythrocytes the electron density is increased $^{24}$ and therefore they appear dark in the reconstructed images. Also, nuclei of the cells can be easily seen, as indicated in Fig. 5c, and the myofiber orientation can be identified. Furthermore, a 3D volume with a rectangular cutoff is depicted in Fig. $5 \mathrm{f}$ in order to visualize the consistent orientation of the myofibers inside the reconstructed volume.

Orientation of cardiac muscle fibers As shown in the last section, cardiac myofibers can be resolved. To quantify the fiber orientation in $2 \mathrm{D}$, a line-detection algorithm based on the Hough transform ${ }^{25,26}$ was used as implemented in MATLAB. The underlying workflow is demonstrated on a single reconstructed slice and summarized in Fig. 6. The first step of the Hough algorithm is using an edge-detection filter, such as a Sobel or Canny filter. In this case, a Canny filter was used and the edge detection threshold was set to an intensity gradient of $20 \%$ between pixels. The result is then a binary representation of the image, as shown in Fig. $6 \mathrm{~b}$. From this binary image, the Hough space was constructed by plotting for each point in this binary image the family of lines that goes through this point. This is done in $\rho, \theta$ - or Hough space, where $\rho$ is the distance of the closest point on the line to the origin and $\theta$ the angle between the horizontal axis and the line connecting the origin with that closest point (see red lines in Fig. 6b). For each point in the binarized image this leads to a unique sinusoidal curve in Hough space (Fig. 6c). In this "sinogram-like" representation lines are represented as bright spots where many curves intersect. The more curves intersect, the more points belong to that specific line. Therefore a threshold can be chosen defining the minimum number of intesections needed to detect a line, which in this case was set to $2 / 3$ of the maximum value. The length and location of each detected line was determined using a corresponding line algorithm. It connects pixels of the binary edge image along the line if the gap between them is smaller than a specified distance, in this case $2 \%$ of the size of the field of view. Lines with a length below $4 \%$ of the size of the field of view were neglected.

This algorithm was applied on all orthogonal slices of the 3D volume and the mean angle and standard deviation of all orientations was calculated. Considering the $x y$-plane, the lines seem to follow very consistently the 

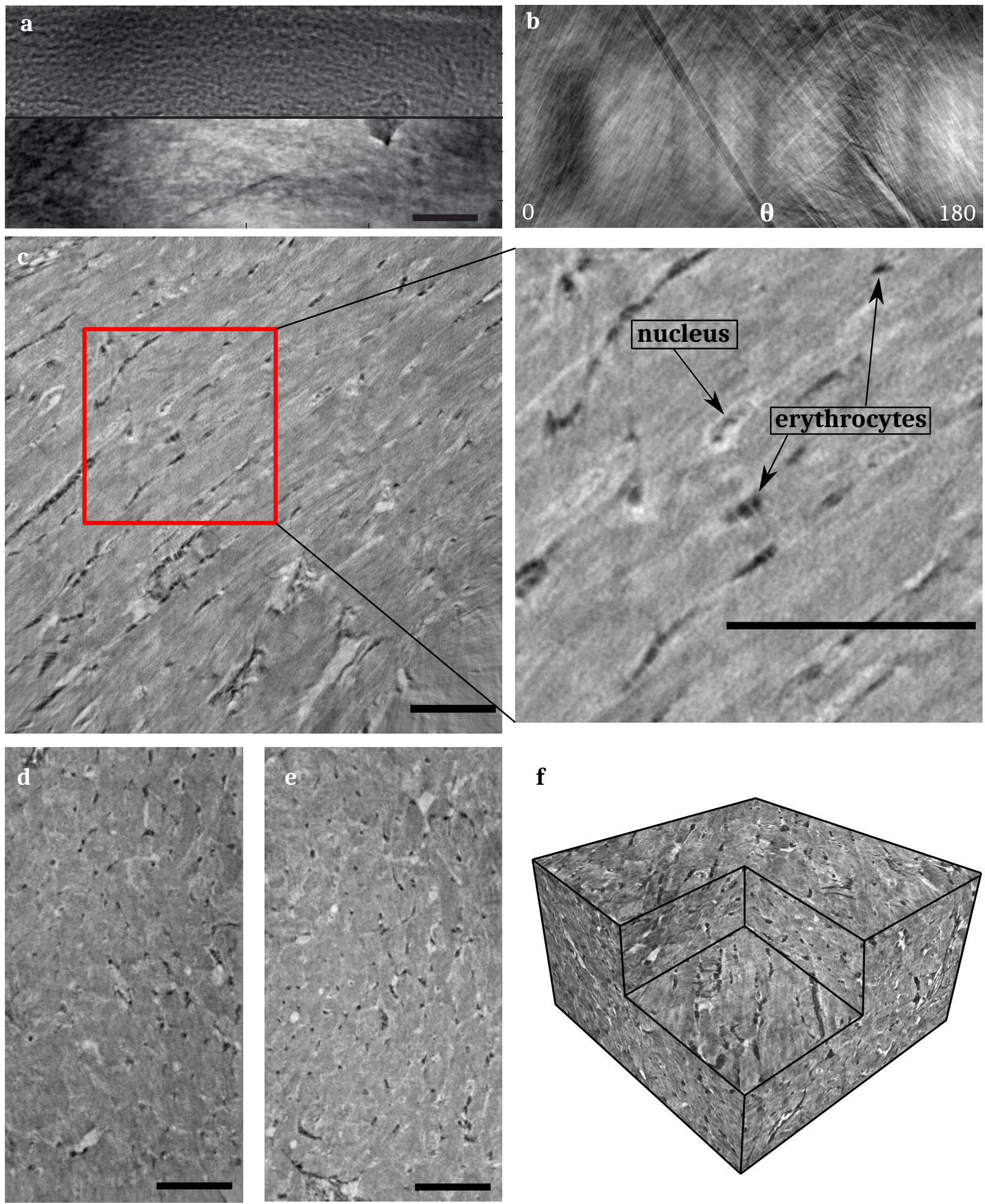

Figure 5. Tomographic reconstruction. (a) An exemplary projection at an acquisition angle of $90^{\circ}$ and a sourceto-sample distance of $150 \mathrm{~mm}$ (top) and the corresponding CTF-reconstruction based on the four recorded distances $x_{1} \in\{145,150,160,180\} \mathrm{mm}$ (below). (b) The sinogram with all reconstructed phase images of 1001 projections recorded over $180^{\circ}$. (c-e) Orthogonal slices through the reconstruction volume. The orientation of the fiber structure is resolved. The scale bar for all images is set to $50 \mu \mathrm{m}$. (f) The rendered 3D volume with a size of $300 \mu \mathrm{m} \times 300 \mu \mathrm{m} \times 150 \mu \mathrm{m}($ Avizo $)$ 

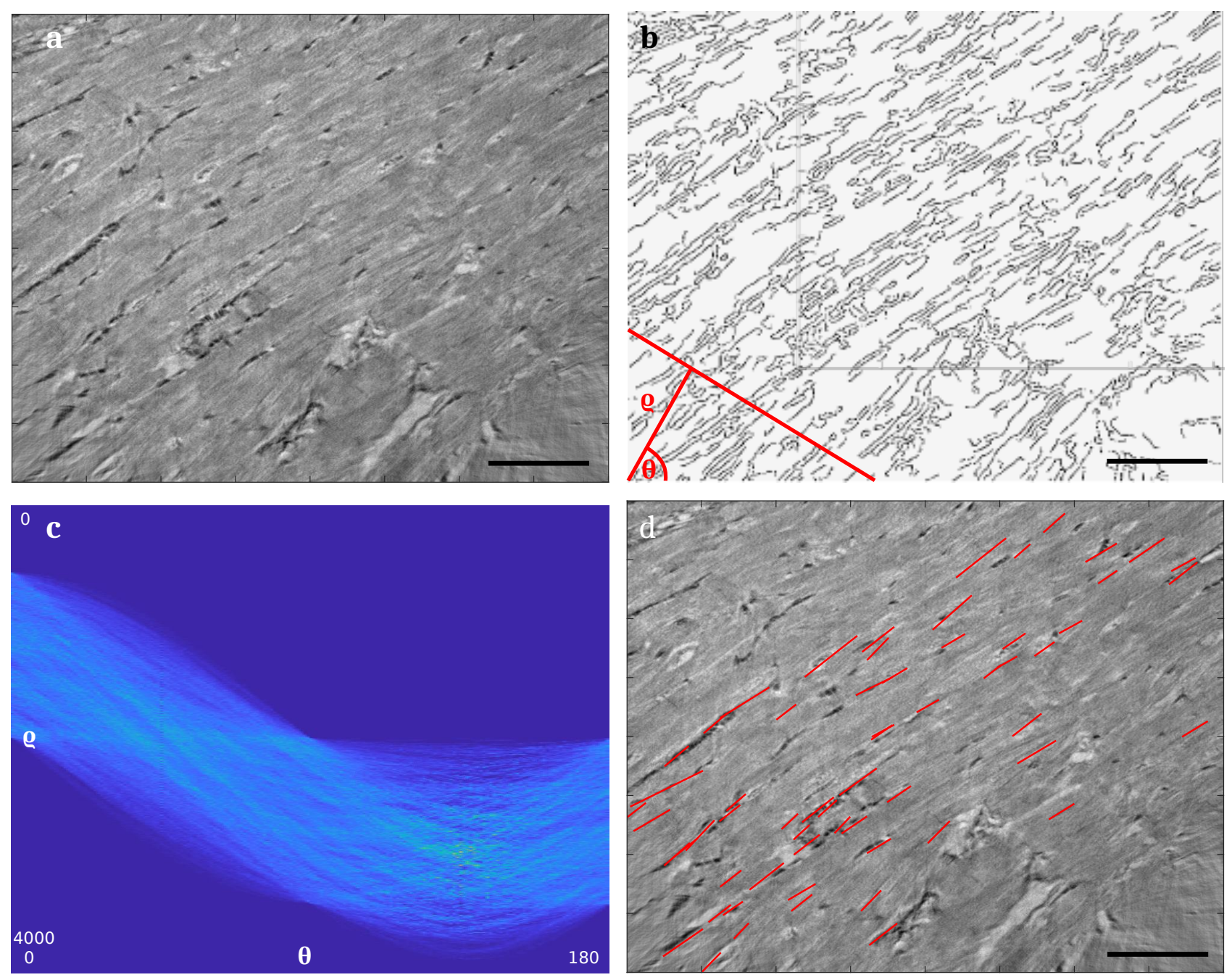

Figure 6. Identification of the myofiber orientation in all transverse slices from the reconstructed volume based on the Hough transform. (a) Exemplary transverse slice. (b) Binarized image after application of a Canny edge filter. (c) Hough space generated from (b). (d) Lines identified from the Hough space are shown in red superimposed on the slice shown in (a), indicating the myofiber orientation. Scale bar: $50 \mu \mathrm{m}$.

direction of the myofibers. The overall angular spread is rather small corresponding to a highly aligned direction of the fibers. We have determined the angle to be at $135^{\circ} \pm 1^{\circ}$. Along the lateral planes, the Hough transform yields incorrect results which can be expected since already from Fig. $5 \mathrm{~d}$ and e it can be recognized that the fibers are cut transversally so that only cross sections of the fibers are seen.

From this it can be concluded that the algorithm identifies the direction of fibers correctly if the reconstructed slice is at least partly parallel to the orientation of the fibers but fails if the fiber axis has an arbitrary orientation. To circumvent this restriction other methods will be exploited in the future to correctly determine the 3D fiber orientation within the volume.

\section{CONCLUSION}

To investigate internal structures of the heart with a non-destructive method, we have used x-ray propagationbased phase-contrast tomography, which is well suited for biological tissue with low absorption. We have successfully combined the tomographic possibilities of a laboratory setup with a synchrotron setup, and, as a result, we were able to deduce the orientation of myofibers at least in one direction. In the high-resolution synchrotron 
experiment, features below the size of the myofibers became visible (erythrocytes, nuclei).

Three different heart tissue samples were screened for quality and intactness at micrometer resolution prior to the synchrotron beamtime. Subsequently a tomogram of the best sample and of a relevant region was recorded at the synchrotron. The structure of the cardiac muscle fibers could be resolved and also erythrocytes and nuclei could be visualized in the small field of view. By applying a Hough line algorithm the $2 \mathrm{D}$ orientation of the myofibers could be identified. In the future, new algorithms will be developed in order to be able to reconstruct the myofiber orientation in 3D. For biological/medical research it would be largely beneficial to obtain a tomogram from the entire heart, e.g. by stitching of several tomograms. Based on the current work, we believe that this will be possible in the future. In the context of cardiac diseases, such studies may provide important 3D structural insight into the cyto-architecture underlying the pathologies.

\section{ACKNOWLEDGMENTS}

We thank Markus Osterhoff and Bastian Hartmann for improvements of the GINIX setup, and Michael Sprung for excellent working conditions at the P10 beamline. Funding by SFB937/A11 and seed funding of the initiative Multiscale Bioimaging of Göttingen Campus is acknowledged.

\section{REFERENCES}

[1] Nicolas, J.-D., Bernhardt, M., Markus, A., Alves, F., Burghammer, M., and Salditt, T., "Scanning x-ray diffraction on cardiac tissue: Automatized data analysis and processing," J. Synchrotron Radiat. 24 (2017). in press.

[2] Salditt, T., Osterhoff, M., Krenkel, M., Wilke, R. N., Priebe, M., Bartels, M., Kalbfleisch, S., and Sprung, M., "Compound focusing mirror and X-ray waveguide optics for coherent imaging and nano-diffraction," $J$. Synchrotron Radiat. 22(4), 867-878 (2015).

[3] Töpperwien, M., Krenkel, M., Müller, K., and Salditt, T., "Phase-contrast tomography of neuronal tissues: from laboratory-to high resolution synchrotron ct," Proc. SPIE 9967, 99670T-99670T (2016).

[4] Bartels, M., Krenkel, M., Cloetens, P., Möbius, W., and Salditt, T., "Myelinated mouse nerves studied by x-ray phase contrast zoom tomography," J. of Structural Biology, 561-568 (2015).

[5] Krenkel, M., Markus, A., Bartels, M., Dullin, C., Alves, F., and Salditt, T., "Phase-contrast zoom tomography reveals precise locations of macrophages in mouse lungs," Sci. Rep. 5, 09973 (2015).

[6] Khimchenko, A., Deyhle, H., Schulz, G., Schweighauser, G., Hench, J., Chicherova, N., Bikis, C., Hieber, S. E., and Müller, B., "Extending two-dimensional histology into the third dimension through conventional micro computed tomography," Neuroimage 139, 26-36 (2016).

[7] Hieber, S. E., Bikis, C., Khimchenko, A., Schweighauser, G., Hench, J., Chicherova, N., Schulz, G., and Müller, B., "Tomographic brain imaging with nucleolar detail and automatic cell counting," Scientific reports 6, 32156 (2016).

[8] Krenkel, M., Cone-beam x-ray phase-contrast tomography for the observation of single cells in whole organs, $\mathrm{PhD}$ thesis, Universität Göttingen (2015).

[9] The MathWorks, I., "Matlab 2017a." Natick, Massachusetts, United States.

[10] Töpperwien, M., Krenkel, M., Vincenz, D., Stöber, F., Oelschlegel, A. M., Goldschmidt, J., and Salditt, T., "Three-dimensional mouse brain cytoarchitecture revealed by laboratory-based x-ray phase-contrast tomography," Sci. Rep. 7, 42847 (2017).

[11] Bartels, M., Hernandez, V. H., Krenkel, M., Moser, T., and Salditt, T., "Phase contrast tomography of the mouse cochlea at microfocus x-ray sources," Appl. Phys. Lett. 103(8), 083703 (2013).

[12] Witte, Y. D., Boone, M., Vlassenbroeck, J., Dierick, M., and Hoorebeke, L. V., "Bronnikov-aided correction for x-ray computed tomography," J. Opt. Soc. Am. A 26(4), 890-894 (2009).

[13] van Aarle, W., Palenstijn, W. J., Beenhouwer, J. D., Altantzis, T., Bals, S., Batenburg, K. J., and Sijbers, J., "The astra toolbox: A platform for advanced algorithm development in electron tomography," Ultramicroscopy 157, $35-47$ (2015).

[14] van Aarle, W., Palenstijn, W. J., Cant, J., Janssens, E., Bleichrodt, F., Dabravolski, A., Beenhouwer, J. D., Batenburg, K. J., and Sijbers, J., "Fast and flexible x-ray tomography using the astra toolbox," Opt. Express 24(22), 25129-25147 (2016). 
[15] Töpperwien, M., Krenkel, M., Quade, F., and Salditt, T., "Laboratory-based x-ray phase-contrast tomography enables 3D virtual histology," Proc. SPIE 9964, 99640I (2016).

[16] Groso, A., Abela, R., and Stampanoni, M., "Implementation of a fast method for high resolution phase contrast tomography," Opt. Express 14(18), 8103-8110 (2006).

[17] Kalbfleisch, S., Neubauer, H., Krüger, S. P., Bartels, M., Osterhoff, M., Mai, D. D., Giewekemeyer, K., Hartmann, B., Sprung, M., and Salditt, T., "The Göttingen Holography Endstation of Beamline P10 at PETRA III/DESY," AIPConf.Proc. 1365(1), 96-99 (2011).

[18] Krüger, S. P., Neubauer, H., Bartels, M., Kalbfleisch, S., Giewekemeyer, K., Wilbrandt, P. J., Sprung, M., and Salditt, T., "Sub-10 $\mathrm{nm}$ beam confinement by X-ray waveguides: design, fabrication and characterization of optical properties," J. Synchrotron Radiat. 19(2), 227-236 (2012).

[19] Homann, C., Hohage, T., Hagemann, J., Robisch, A.-L., and Salditt, T., "Validity of the empty-beam correction in near-field imaging," Phys. Rev. A 91, 013821 (2015).

[20] Zabler, S., Cloetens, P., Guigay, J.-P., Baruchel, J., and Schlenker, M., "Optimization of phase contrast imaging using hard x rays," Rev. Sci. Instrum. 76(7), 073705 (2005).

[21] Cloetens, P., Ludwig, W., Baruchel, J., Van Dyck, D., Van Landuyt, J., Guigay, J. P., and Schlenker, M., "Holotomography: Quantitative phase tomography with micrometer resolution using hard synchrotron radiation x rays," Appl. Phys. Lett. 75(19), 2912-2914 (1999).

[22] Turner, L., Dhal, B., Hayes, J., Mancuso, A., Nugent, K., Paterson, D., Scholten, R., Tran, C., and Peele, A., "X-ray phase imaging: Demonstration of extended conditions for homogeneous objects," Opt. Express 12(13), 2960-2965 (2004).

[23] Ketcham, R. A., "New algorithms for ring artifact removal," Proc. SPIE 6318, 63180O-63180O-7 (2006).

[24] Reifenrath, L. R. S., Ultrastrukturelle und glykohistochemische Untersuchungen am Blut der Katze (Felis catus), PhD thesis, lmu (2011).

[25] Hough, P. V. C., "Method and means for recognizing complex patterns." US Patent 3,069,654 (1962).

[26] Illingworth, J. and Kittler, J., "A survey of the hough transform," Computer Vision, Graphics, and Image Processing 44(1), 87 - 116 (1988). 